\title{
Ueber die Nervensubstanz in einem soliden Overial=Teratom.
}

\author{
Von \\ Ichiro Maruyama. \\ Aus der Fraın'klinik der medizinischen Fakultät zu Okujama. \\ (Vorstand: Prof. Dr. K: Ando) \\ Eingegungen «m 14. Muj 1927.
}

Die Teratomen entwickeln sich hauptsächlich aus den Geschlechtsdrüsen und werden nach ihren klinischen und morphologischen Verhalten in zwei Gruppen, von cystischen und soliden geteilt. Aber die beiden haben densellben Ursprungen u. z. sie entwickeln sich aus zwei oder drei Keimblättern (Heijel).

Die cystischen Teratomen sind von fertigen Geweben zusammengesetzt und die neisten Autoren nemnen sie Missbildungen wegen ibrer klinischen Gutartigkeit. Die soliden Teratomen sind dagegen von embryonalen Geweben zusammengesetzt und betragen den malignen Character, sodass sie als wirkliche Neubildungen oder maligne Geschwülste beobachtet werden.

Was die Nervensubstanz in soliden Teratomen betrifft, so befindet man in der Regel die centralen Nervensubstanzen. Die betreffenden Nervensubstanzen bestehen aus den zu embryonalen nnd reifen Stadien zugehörigen physiologischen Geweben, wenngleich sie die pathologischen Erscheinungen in malignen Fällen aufweisen.

1ch habe im Juli 1923 in der hiesigen Frauenklinik zufälligerweise einen echten im rechten Ovarium entwickelten mannskopfgrossen 1260 gr. wiegenden Teratom beobachtet. Die allgemeinen histologischen Befunde wurden schon in "Okayama-Igakkai-Zassi , im August 1925 veröffentlicht, Infolgedesseu werde ich hier hauptsächlich die Nervensubstanzen betreftenden Befunde anführen.

\section{Literatur.}

Im 1896 hut Otto Gaell vorstüdiges Fehlen des Nervengewebes im intruljgamentär entwickelten Terutom ungegeben. Fulk (1900) but die grossen und kleinen pymmidenförmigen multilocularen Gunglienzellen in eivem Ovariul-terıtom mit Metıstusierung konstıtiert. Glcckner (1901) hıt pyrımidenförmige Gunglienzellen, mit Cylinderepitbel ausgekleideten Ventrikel, Centralciunuanlige mit Plexus chorioidej, 
Bündel von marklosen Nervenfusern, Choriojdalpigment und Retinn beobachtet. Neubiser (1906) hat die beiderseitigen Ovariultemtomen untersuoht und das Vorbundensein von centralem Nervensystem mit embryonnlem Chrncter, Neuroepithe], Glin, Ganglienzellen und Nervenfasern bestïtigt. Pfannenstiel (1908) hat 7 solide Ovarialterutomen zusnmmengestellt und hat auf dus Vorhandensein von grossen und kleinen Inseln der Gehirnmasse, welche den ersten Fötulmonaten entsprechenden Bau aufweisen, in der Nihe von Knorpelstiicken und Knorpelinseln aufmerksam gemacht. In der gennnnten Gehiımasse hat der Vertnsser den Plexus, chorioidei mit nervöser Substanz, Chorioidalzellen mit plumpem embryonalem Bun, Streifen von tiefsehwnrzem Pigmente, uus sechseckigen Pigmentepitheln bestehenden Ventrikel, einen primären Augenbecher mit äusse: em Blutt aus typischer Lamina pigmentosa retinne und innerem Blatt aus embryonaler geschlängerter Retinn, Ganglienzellen, Neuroglia mit varikösen Nervenfasern. Rosenstein (1913) hat bei 15 jührigem Mädchen in ejnem mit rechtsseitigem mannskopfgrossem Ovarinldermoid combinierten Kindsfuustgrossen Ovarinlteratom grosse Menge von Gebirnsubstanz mit Chorioidea gefunden. Winkler (1914) hat bei ejnem nengeborenen Madohen 7 Tage post Purtum einen intruperitonenl entwickelten Teratom gefunden und hat in ihm eine mit Zentralnervensubstanz umgebende schädelkapsel ahbnliche Bildung konstatiert. Matsui (1915) bat in einem krebsig entarteten, jm dem Blinddarm hervorragenden rechtsseitigen Ovarialteratom Neuroglin, Nervenzellen, Neuroepithel, Hirnsnnd, Hirnhäute mit Pigmentepthelien, Spinnlganglien und perephere Nervenfuser nuchgewiesen. Ono und Taknoka (1917) hat in einem Tubenembryom Hingewebe (Nerven- und G]inzellen), Hiınventrikel (ein Wundtheil dnvon scheint sjoh um Chorioiden zu haudeln), Ganglien, Spinnlnervenfuserbündel konstatiert. Kaneko (1924) bat bei einem mïnnlichen Fötus vom zebnten Schwnngerschaftsmonat in einem retroperitonealterntom Hiınhiute, Chorioiden, Ependiumzellen, Gnnglienzellen und ncch nicht git ausgebildete Huutnerven au fgefunden.

Als pathologische Gewebe haben Rosenstein (1898) und Saxer (1902) einen Fall von Neuroepitheliom, welcher aus medullaren Zellen bestehen, mitgeteilt. Finkelnburg hat im Hirngewebe die Cancer-gebilde, welche von Ependiumzellen ausgegangen zu sein scheint, gefunden. Backhaus (1901) nnd Suxer (1902) hnben die zylinderzellencarzinomahnliche Zellmasse im peripheren Anteil von ejnem Ovarialteratom konstutiert. Risel (1904) and Sjöval (1911) haben die syncytiılen Elemente in Neuroglin beobachtet. Schwalbe bat die :ihnlichen Befunde von Bcckhaus-Saxer's Fall in solidem Anteil von einem cystiech-soliden Ovarialteratom gefunden. Rubbert (1914) hat ins Adenosurkom umgewandelte Zentralnervensubstanz in einer Nierenterntoidgeschwulst gesehen. Hejjel (1922) hat cnncerahnliches Gewebe in Zentrulnervensubstunz von einem Tentom anfgefunden. Auch hat er die drüsenähnliche Formationen, welche dichtzellig, sarcomähnlich und labyrinthartig sind, im Glingewebe ein Hcdenteratom; im Tumorbuch typische Untersuchung von vielen Plexusformationen beobachtet. Weiter hat er bei Untersuchuug von vielen acurdiulen Zwillingsmissgeburten die Adenosarcom-, Rundzellensarcom-, Gliosarcom-, polymorphzellensaicom-, Adenocarzinom:ihnliche Gebilde in Neuroglin aufgewiesen.

\section{Histologische Befunde.}

Hier verzichte ich auf die Beschreibung der Untersuchungsmethcdik, weil ich sie scbon in anderer Dissertution “ Ueber einen Full von solidom Ovuriulterntom " geauu besch ieben habe.

Das Nervengewebe entwickelt sich mit der Haut vom Ektoderm. Die im Overial- 
teratom sich vortindenden Nervengewebe weisen wie andere Gewebe verschiedene Entwickelungsphasen von embryonaler bis zur reifen auf und grösstenteils bestehen sie aus den embryonalen Typen, welche im Tumor als grosse und kleine Klampen disseminieren oder mit anderen Geweben vermischt sind (fig. 1. und 2). Die von mir aufgefundenen Nervengebilde sind: Hirngewebe, Gliagewebe, Plexus chorioidei, Chorioidealzotten, Nervenzellen, Neuroepithel, Hịnhäute mit Pigmentzellen, Spinarganglienzellen (fig. 3), sympathische Ganglienzellen, markhaltige und marklose Nervenfasern und noch nicht gut ausgebildete periphere Nerven in Haut und Muskel. Als pathologische Gebilde habe ich rundzellensarcomähnlichen Herd in Neuroglia beobachtet (Fig. 4).

Der durch meine micro-chemische Untersuchung aufgehobene merkwürdige Befund ist die Ur-anlage. Inder Figur 5 sieht man eine Anlage, welche aus den ein Lumen begrenzenden jungen Nervenzellen bestehen. Dieselben jungen spixdelformigenzellen sind alle fast gleichartig gebaut und ordnen sich radiär gegen das Lumen an. Sie sind bipolar u. z. sie haben lange obere und untere Fortsätze. Die Dimensionen dieser Fortsätze sind alle fast gleich obwohl geringe Sehwankungen sich zeigen. Es ist schwer festzustellen ob diese Zellen Glioblasten oder Neuroblasten sind, trotzdem sie ganz sicher Nervenzellen sind. Innerhalb des Zellverbandes befindet sich eingekeilt einige grosse runde Zellen, welche an Ganglienzellen erinnern und vielleicht zu Astrocyten gehoren.

Aus oben angeführten Befunden scheint dieses Gewebe nicht ausgebildetem Nervengewebe anzugehoren, sondern es scheint sich um Ur-anlage von demselben zu handeln. Durch Nissel's Färbungsmethode kann ich einige Gruppierungen von 4-6 Zellen in der Nähe von Zellenkomplex, welcher den Randtheil des Neurorolıres andeutet, konstatieren. Diese Zellen sind durch Nissl's Methode als Ganglienzellen bewiesen.

In einem Teil des Tumores sieht man einige Anhäufungen von den Ganglienzellenleisten. Hier sieht man nur Haufen von kleinen runden Kornern, infolgedessen sind die Ganglien- und Stützzellen scher zu unterschieden (Fig. 6). Auch sieht man Zellen. gruppierungen von 1-2 Kernen enthaltende protoplasma reiche Zellen, welche etwas fortgeschrittenes Stadium anweisen. Das ganze Bild stellt eben die Ganglien-und Kapselzellen der. Die Zellgrenzen der Kapsekzellen sind sehr undeutlich und zeigen das Aussehen von Syncythium (Fig. 7 ).

Aus den oben dargestellten Ergebnissen kann ich die betreffenden Nervenzellen als solche Kapseltragenden Nervenelemente betrachten, welche sich noch im früheren Embryonalstadium befinden und in welchen Sympathicus-und Spinalganglienzellen sind, noch nicht differenziert werden.

\section{Schlussfolgerung.}

1) Das Nervengewebe, das in einem Ovarialteratom sich vorfindet, besteht aus den 
Nervengeweben, welche den verschiedenen Entwickelungsstufen entsprechend die verschieden gradig entwicklten Zellen von embryonaler bis zur reifen enthalten. Die embryonalen überwiegen bedeudend an Zahl.

2) Im oben genannten Nervengewebe befindet sich maligne Entartung wie in anderen Teratomen.

Znm schluss dieser Dissertation bin jch sehr verpflichtet mejnen herzlichen Dhınk für freundliche Anleitung und R.tgebung von Herrn Prof. Dr. Ando unszusprechen. Gleichzeitig spreche ich nuch grossen Dank für peinlichste Durchmusternng der vielen microskopischen Präparaten und frenndliche Rutgebung von Herrn Prof. Dr. Kosaka, Seki (nn hiesigem anat. Institnt), Tiımnn (nn hiesigem path, Inst,tut), Huyashi (un besigem peychint. Instjtnt), und K. Adnchi.

\section{Literatur.}

1) Askunizy, Dentsch. Gesellsch. 1907.

2) Buckhatns, Aıcbive f. Gy: Br. 631901

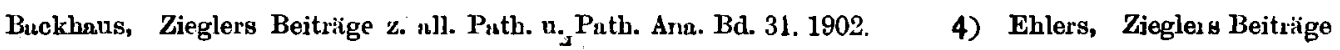
z. ull. Puth. u. Puth. Anu. Bd. 55. $1913 . \quad$ 5) Fulk, Monut. f. Geb. u. Gy. Bd. 12. 1900. 6) Gsell, Aıchiv f. G.. Bd. 51. $1896 . \quad$ 7). Glcekne, Zent $t$ nl. f. Gy. Bd. 25. $1901 . \quad$ 8) Hejjl, Virchows Aıcliv f. Puth. Anı. u. Physijl. Bd. 229. 1921. 9) Heijl, All. Path. u. Prth. d. Mensclen u. d. Tiere. 1922. 10) Kıneko, Nippon-Fuzinkngıkkaizıssi. 1023 . 11) Maruyamn, Okaynma-Igakkaiznss.i 1924. 12) Mnzuj, Hoknetu-Igakkaizassi. 1925. 13) Mayer, Bundbuch d. Biologie u. Pathogie des Weibes. Bd. 5. 1926 14) Nenhiuser, Aichiv f. Gy. Bd. 79. $1906 . \quad$ 15) Ohno-Tuknokn, Nippon-Fuzinkıgıkkunzıssi. Bd. 79. 1906. 16) Pfunuenstrel, Veit's Hindbuch der Gynukologie. Bd. 3. 1908 17) Ribbert, Geschwulstlehre. Bonn. 1914 . 18) Rosenstein, Zentrul. f. Gy. Bd. 37. H. 2. 1913 . 19） Rosenthial, Zieglers Beitrïge z. all Puth. u. Puth. Anı. Bd. 23. 1898 20) Sıxer, Zieglers Beitrige \%. all. Path. n. Path. Anu. Bd. 32. 1902.21 2) Suxer, Zieglers Beitrige z. all. Path. u. Path. Anu. Bd. 20. 1896. 22) Sixer, Zieglers Beitrüge z. all. Path. u. Path. Ana. Bd. 31. 1902. 23) Sizer. Archiv f. Gy. Bd. 63.1901. 24) Sklıwunos, Vi.chows Archiv f. Path. Ana. u. Physiol. Bd. 232. 1921. 25) Schwalbe, Frankfurter Zeit. Path. 1911. 26) Sjô̄ıll, Fraukfiurter Zeit. f. Path. 1911. 27) Winkler, Stnd. z. Putho. d. Entwickel. Pd. 1. H. 2.1914.

\section{Erklärung der Abbildungen.}

Fig. 1. Embryomuler Nervensubstunz.

Fig. 2. Reife Nervensubstunz.

Fig. 3. Spindergınllienzellen.

Fig. 4. Rnndzellensurcomähnliches Herd in Neuroglin.
Fig. 5. Ur-nnlnge des Nerrengewebe.

Fig. 6. Anhaufungen von den Ganglienzellenleisten.

Fig. 7. 1. Gungljenzellen.

2. Kupselzellen. 


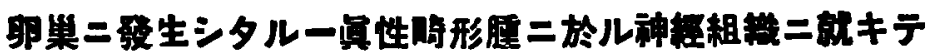

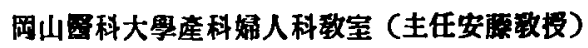

丸山 $\rightarrow$ 郎

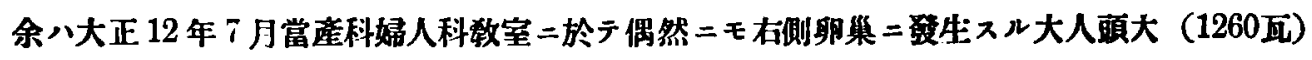

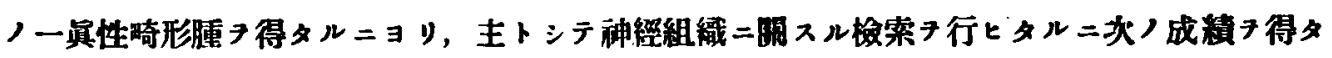
y.

1. 卵巢猗形腫中二存在セル神經祖織八, 胎生初期ノモノョリ成整セルモノニ至ルマデノ, 各

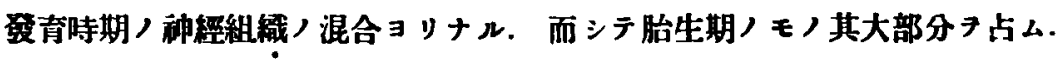

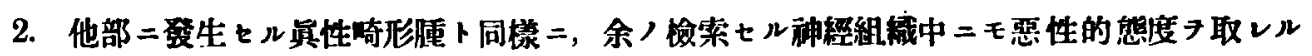

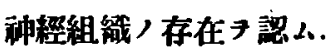


Fig. 1.

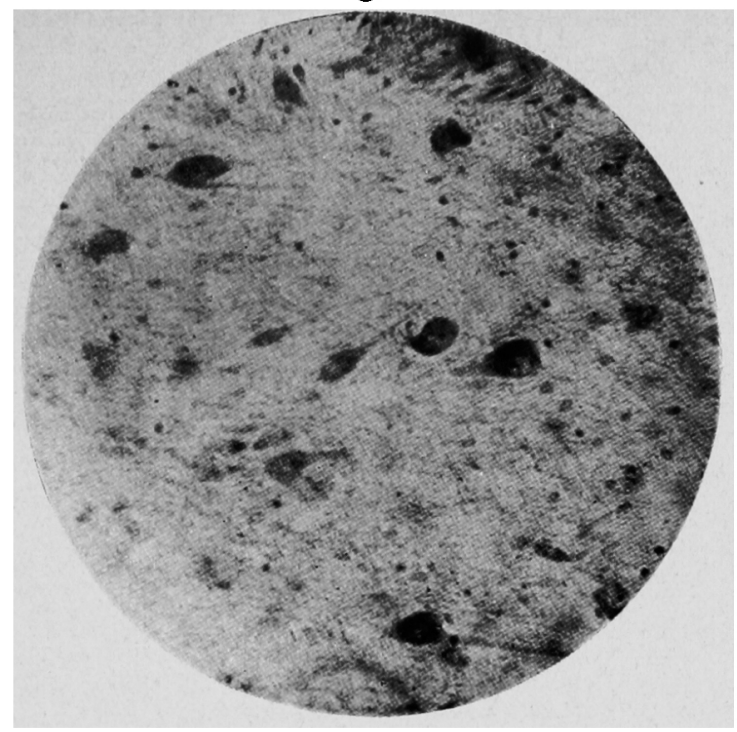

Fig. 2.

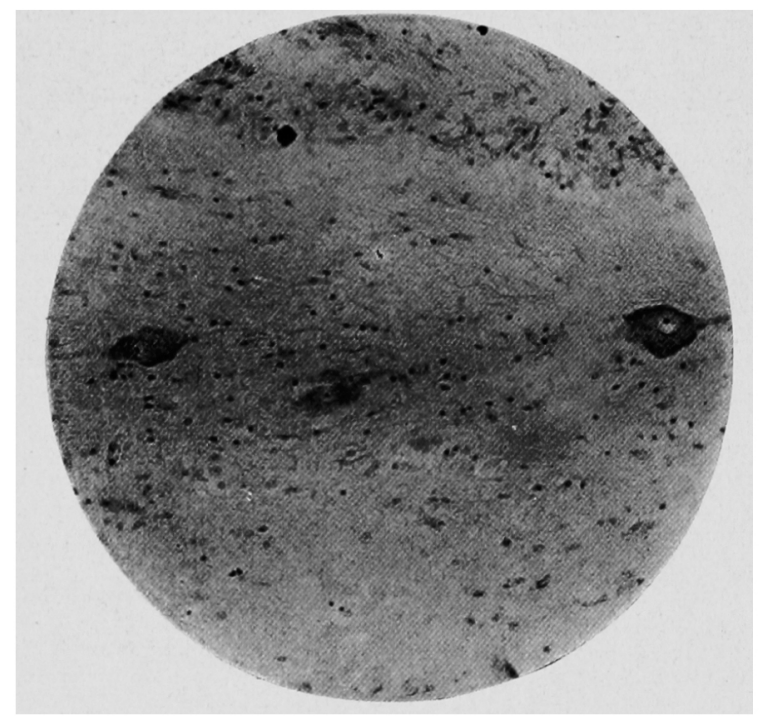

Fig. 3.

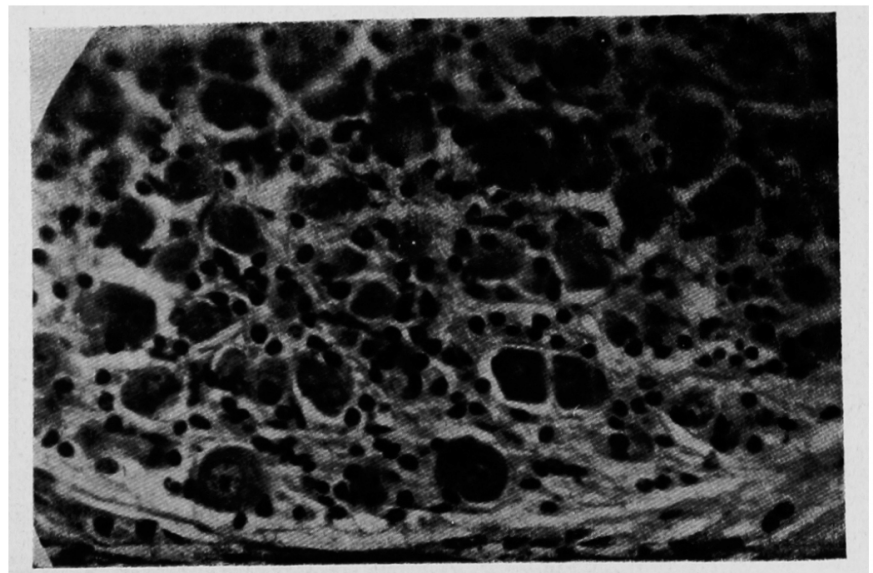

Ichiro Maruyama-Ueber die Nervensubstanz in einem soliden Orarin Tricm. 
Fig. 4.

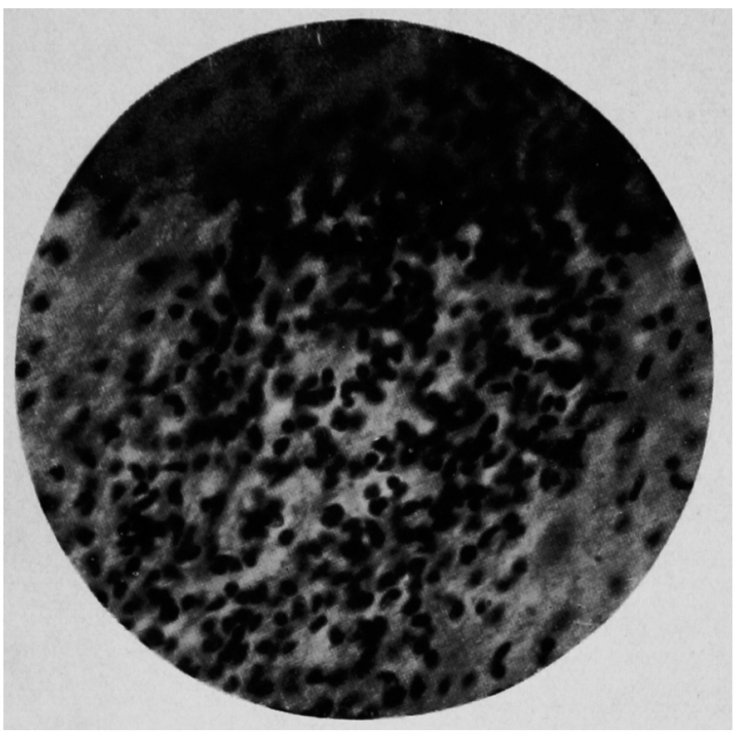

Fig. 5.

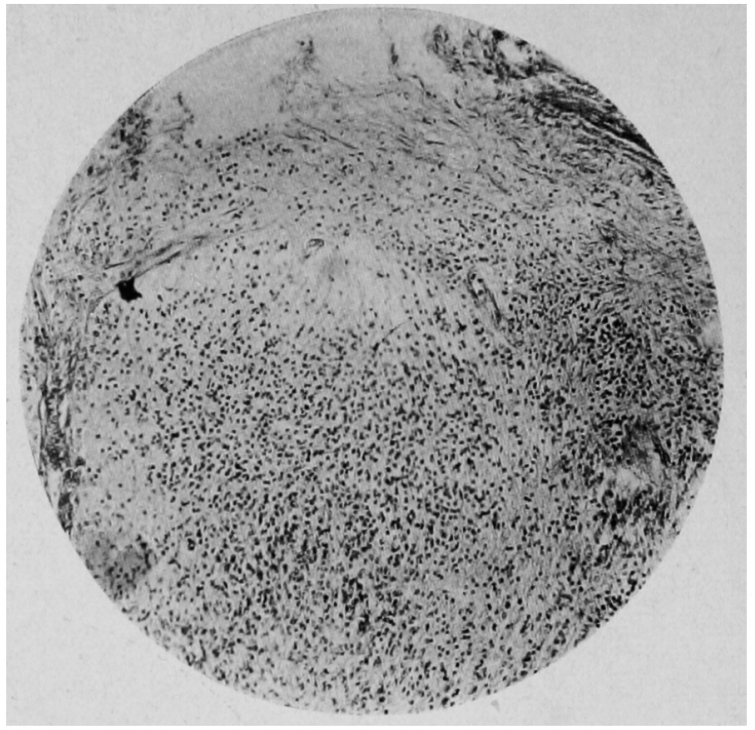

Fig. 6.

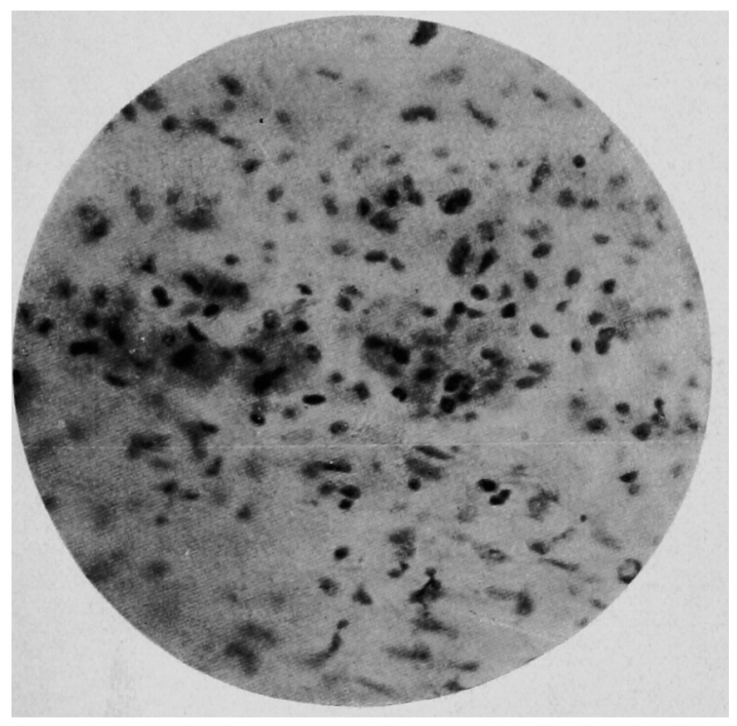

Fig. 7.

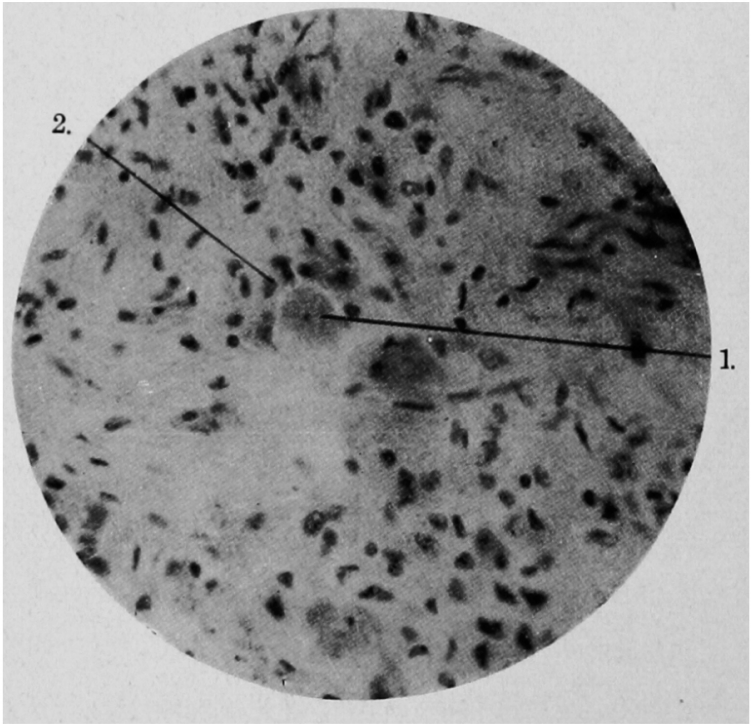

Ichiro Maruyama-Ueber die Nervensubstanz in einem soliden Ovarial-Teratom. 